\title{
Préhistoires Méditerranéennes
}

$10-11$ | 2002

Varia

\section{Les industries lithiques du Chasséen en Languedoc oriental : caractérisation par l'analyse technologique}

Résumé de thèse par l'auteur. Université de Provence, juin 2002, 466 p., 73 pl.h.t.

\section{Vanessa Léa}

\section{OpenEdition}

\section{Journals}

Édition électronique

URL : https://journals.openedition.org/pm/281

DOI : 10.4000/pm.281

ISSN : 2105-2565

\section{Éditeur}

Association pour la promotion de la préhistoire et de l'anthropologie méditerranéennes

Édition imprimée

Date de publication : 1 septembre 2002

Pagination : 227-231

ISSN : 2118-8211

\section{Référence électronique}

Vanessa Léa, « Les industries lithiques du Chasséen en Languedoc oriental : caractérisation par l'analyse technologique », Préhistoires Méditerranéennes [En ligne], 10-11 | 2002, mis en ligne le 23 avril 2009, consulté le 08 avril 2022. URL : http://journals.openedition.org/pm/281 ; DOI : https://doi.org/ $10.4000 / p m .281$

Ce document a été généré automatiquement le 8 avril 2022.

Tous droits réservés 


\section{Les industries lithiques du Chasséen en Languedoc oriental : caractérisation par l'analyse technologique}

Résumé de thèse par l'auteur. Université de Provence, juin 2002, 466 p., 73 pl.h.t.

Vanessa Léa

\section{Objectifs et cadre de l'étude}

1 Cette thèse se proposait d'analyser la variabilité des assemblages lithiques du Chasséen (4500 - 3500 BC. Cal.) en Languedoc oriental. Cette région, extrêmement bien documentée pour le Chasséen, n'avait jusqu'à présent pas fait l'objet d'études lithiques. Un travail de fond sur cette aire géographique répondait donc à une lacune, et a de plus permis une mise en relation entre les données provençales (Binder 1984, 1991 ; Binder \& Gassin 1988 ; Léa 1997) et celles du Languedoc occidental (Briois 1997 ; Vaquer 1990).

2 Cette analyse s'intéresse pour l'essentiel aux productions sur silex blonds bédouliens $d u$ Vaucluse débités par pression, qui composent une proportion en général très importante des assemblages lithiques chasséens. Or, ces productions sont de précieux révélateurs sociaux. La diffusion des silex bédouliens, depuis le Vaucluse, de par son ampleur géographique - tout le Midi de la France jusqu'à hauteur de Lyon, Catalogne à l'ouest et Piémont-Ligurie à l'est - son abondance et sa diversité dans les modalités de circulation, constitue une approche privilégiée des sociétés du Chasséen méridional. En effet, la chaîne opératoire des silex bédouliens chauffés, éclatée en différents lieux, illustre à elle seule une structuration complexe de l'espace et de la société et a été un élément important dans la formulation de l'hypothèse du développement de l'artisanat au Chasséen (Binder \& Perlès 1990). 
L'analyse est ici guidée par plusieurs axes de réflexion :

- Chronologie :

- Les variabilités stylistiques identifiées en Provence se retrouvent-elles en Languedoc oriental?

- Les variabilités stylistiques sont-elles exclusivement interprétables en terme de chronologie?

- Géographie :

- Existe-t-il des particularités technologiques ou typologiques spécifiques au Languedoc oriental?

- La variabilité des assemblages est-elle la même que celle connue en Provence ? Comment circulent les matériaux provençaux (bédouliens et rubanés) vers l'ouest ? Circulent-ils sous les mêmes formes et dans les mêmes proportions que ce qui est attesté en Provence? Enfin, font-ils l'objet des mêmes gestions?

- Structuration sociale et spatiale :

- L'hypothèse de la répartition sociale des tâches trouve-t-elle ici des arguments?

- Hormis la circulation sur de grandes distances, comment l'industrie lithique peut-elle contribuer à la mise en évidence de la complémentarité existant entre sites?

- Que peut-on connaître des rythmes de production? Les nucleus sont-ils débités en une seule fois, ou bien en des temps différents?

\section{Composition du mémoire et démarche}

4 Cette recherche, s'articule autour de trois parties qui totalisent quatorze chapitres. Il était dans un premier temps nécessaire de reprendre succinctement et partiellement les problématiques actuelles relatives au Chasséen et plus particulièrement en Languedoc oriental (Léa 1998). Ceci a permis de définir précisément les questions auxquelles l'analyse technologique devait répondre, avant d'expliciter la démarche suivie (chapitres I à III). Les résultats sont ensuite exposés de manière monographique afin de conclure sur les aspects spécifiques à chaque site (chapitres IV à XI). Enfin, différentes interprétations sont proposées et discutées (chapitres XII à XIV). Chaque hypothèse est testée (qu'elle soit d'ordre chronologique, géographique ou social...) non seulement à partir du corpus du Languedoc oriental, mais aussi en faisant la liaison avec les données provençales et du Languedoc occidental, permettant alors une vision plus large de la diffusion des silex bédouliens, depuis le Vaucluse jusqu'à la Catalogne.

5 Le corpus est composé de huit études monographiques auxquelles il faut ajouter sept sites partiellement étudiés et intégrés en synthèse. Ces assemblages sont traités inégalement selon leur potentiel informatif. Ils sont présentés selon la phase chronologique à laquelle ils appartiennent :

1. la phase récente du Chasséen: Lattes (Lattes, Hérault), la Ramière (Roquemaure du Gard, Gard), Jarnègues (Jonquières-Saint-Vincent, Gard), Le Réal (Montfrin, Gard) ;

2. les phases anciennes du Chasséen : Port-Ariane (Lattes, Hérault), Le Crès (Béziers, Hérault)

3. les sites à chronologie mixte ou interne: Raffègues (Mèze, Hérault) et la Madeleine (Villeneuve-les-Maguelonnes, Hérault).

6 La démarche suivie est celle de la technologie lithique. Une méthodologie déjà élaborée et spécifiquement adaptée au débitage laminaire par pression en contexte chasséen (Léa 1997), a de même été utilisée. 


\section{Apport de l'étude}

7 L'appréciation de la variabilité des assemblages et du débitage laminaire sur silex bédouliens chauffés, a mené à la proposition d'hypothèses d'ordre chronologique, géographique, fonctionnel ou social.

\section{Variabilité des assemblages et du débitage laminaire}

8 La variabilité des assemblages et du débitage laminaire a été appréciée à différentes échelles : entre sites, entre différentes productions d'un même assemblage, au sein des productions en silex bédouliens. Ces comparaisons ont été réalisées en respectant les phases chronologiques du corpus: phases récentes, phases anciennes et sites à chronologie mixte ou interne.

9 - La variabilité de la composition des assemblages a été étudiée selon la proportion des différents matériaux au sein de chacun d'eux, et selon celle des silex bédouliens chauffés et non chauffés.

10 - La variabilité du débitage laminaire sur silex bédouliens a montré des disparités dans les modalités de circulation, tant pour les silex non chauffés, qui diffusent sous forme de produits finis, que pour les préformes en silex traités thermiquement. Le débitage de lamelles par pression sur préformes chauffées a fait l'objet d'une attention particulière. Chaque catégorie technologique de lamelles a été comparée d'un site à l'autre afin de caractériser la variabilité des modalités de la mise en forme, du débitage, et de la réfection (type de plan de pression, traitement de la corniche, parallélisme des nervures, déroulé de la lamelle...).

11 - La synthèse des données sur l'outillage a mis en évidence une certaine diversité, notamment en ce qui concerne les armatures. De ce point de vue, l'un des résultats les plus notables est sans doute la minorité des trapèzes sur lamelles, souvent considérés comme marqueurs du Chasséen méridional.

\section{Les interprétations géographique et chronologique et leurs limites}

12 L'observation de ces variabilités a mis en relief des résultats contrastés dont il a fallu comprendre la signification. Les hypothèses géographique et chronologique ont donc été testées.

\section{L'hypothèse géographique}

13 - L'hypothèse géographique a été envisagée, dans un premier temps, en étudiant la diffusion des matériaux provençaux (rubanés et bédouliens) vers l'ouest. Cette observation a mis en évidence une raréfaction des silex rubanés en Languedoc par rapport à la Provence où ils sont bien représentés notamment dans les phases récentes du Chasséen, et des modalités de circulation différentes pour les silex bédouliens : les lames non chauffées qui circulent sous forme de produits finis comportent plus souvent des traces de mise en forme en Languedoc qu'en Provence et les techniques de taille montrent une importante diversité, signant sans doute là une position de périphérie par rapport à la zone nucléaire provençale. 
14 - Dans un deuxième temps, l'influence provençale en Languedoc oriental a été mise en relief à travers l'étude des armatures : les pointes bifaciales y sont en effet relativement bien attestées, tout comme en Provence, et contrairement aux contextes chasséens languedociens connus.

\section{L'hypothèse chronologique}

15 - La phase proto-chasséenne, caractérisée pour la première fois en Provence (Giribaldi, Nice ; Binder 1996) a trouvé quelques ressemblances dans l'assemblage du site du Crès (Béziers, Hérault). Néanmoins, les produits laminaires en silex bédouliens non chauffés importés sont plus irréguliers. De plus, un débitage original d'éclats sur éclat en silex bédouliens importés atteste l'existence d'une tradition technologique du Néolithique ancien rhodano-provençal (Gassin \&Binder, sous presse).

16 - En Provence, les styles de débitage attestés sur silex bédouliens chauffés, révèlent des phases chronologiques différentes : débitage de type semi-conique du Chasséen ancien, et débitage de type quadrangulaire plat du Chasséen récent (Binder 1991). En Languedoc oriental, le style de débitage de type Chasséen ancien n'a pour l'instant pas été clairement identifié. La reprise des données disponibles sur les assemblages lithiques du Languedoc occidental a montré que la situation semblait identique plus vers l'ouest. La circulation de préformes chauffées en Languedoc pourrait donc s'effectuer avec un temps de retard par rapport à la Provence, à un moment où les préformes semi-coniques circulent déjà associées à des nucleus quadrangulaires plats. Le style proto-chasséen du Languedoc serait donc en partie contemporain du style chasséen ancien de Provence. Nous serions ainsi face à un phénomène d'inertie culturelle déjà remarqué pour la néolithisation du Languedoc occidental (Vaquer 1989).

17 - Notre étude a enfin montré que la variabilité du débitage laminaire n'était pas seulement interprétable en terme de chronologie (Binder 1991). En effet, l'analyse des chaînes opératoires sur silex bédouliens chauffés a démontré qu'il existait des modalités de passage entre un style quadrangulaire plat et semi-conique. Une préforme peut donc être débitée en fin de production selon un style différent de celui de départ. Cette transformation du bloc de départ n'a cependant pas toujours lieu. Il y aurait donc différentes façons de faire selon les sites.

\section{Implications sociales, fonctionnelles et culturelles}

18 Si les hypothèses géographiques et chronologiques ne suffisent pas à interpréter la variabilité du débitage laminaire au Chasséen, il a donc fallu proposer d'autres explications. Des arguments d'ordre social, fonctionnel ou culturel ont donc été envisagés.

\section{Silex bédouliens et savoir-faire}

19 L'analyse des productions en silex bédouliens a permis de poser l'hypothèse de savoirfaire inégaux entre producteurs spécialisés et consommateurs dont le savoir-faire serait moindre. L'un des intérêts premiers du Languedoc oriental est de présenter une source d'approvisionnement locale de silex blonds bédouliens : les Costières du Gard renferment dans les anciennes terrasses rhodano-duranciennes de nombreux galets de 
silex bédouliens arrivés depuis le Vaucluse par apport fluviatile (Bazile, sous presse). Ces galets constituent donc ici, de manière originale, une source potentiellement exploitable pour un débitage de lamelles par pression. Or, ils ont seulement été employés pour un débitage expédient d'éclats au même titre que n'importe quelle autre matière première locale (débitage d'éclats irréguliers rapidement réalisés donc peu investis techniquement). Parallèlement, les sites du Languedoc oriental montrent, sur les sites consommateurs, des importations intensives depuis le Vaucluse de préformes en silex bédouliens, destinées à la production de lamelles. Pourtant les galets locaux de silex bédouliens (de module important et rarement micro-faillés), présentaient les mêmes aptitudes à la taille que les blocs préformés et importés par main d'homme. Ils auraient donc parfaitement convenu au débitage de lamelles par pression, comme plusieurs expérimentations de taille l'ont d'ailleurs démontré. Cette situation traduit donc bien des savoir-faire différents : les Chasséens de cette région ne savaient sans doute pas mettre en forme ces galets pour un débitage par pression.

\section{Silex bédouliens et complémentarité entre sites}

L'étude des réseaux de diffusion en Languedoc oriental a permis de mettre en évidence des disparités dans les modes de distribution des silex bédouliens, ainsi que dans les manières de les débiter :

1. Statut des sites. Certains sites du Languedoc, moins bien alimentés en silex bédouliens, semblent en marge des réseaux de diffusion. La proportion de silex bédouliens n'est pourtant pas inversement proportionnelle à la distance parcourue depuis les sources de matières premières en Vaucluse. De telles disparités, dans les quantités et les modalités de circulation, révèlent ainsi des différenciations dans le statut des sites (Briois \& Léa, sous presse).

2. Rythmes d'occupation et complémentarité entre sites.Parmi les différents éléments qui circulent(produits finis: lames et éclats ; ou préformes à tailler sur les sites consommateurs), les préformes sont particulièrement riches d'informations. L'analyse des assemblages lithiques a en effet montré que les préformes importées sont parfois débitées en différents temps et en différents lieux, sur plusieurs sites consommateurs. Elles constituent ainsi une réserve que l'on garde avec soi pour répondre à des besoins ponctuels, lors de déplacements au sein du terroir. Cette modalité d'exploitation des préformes révèle sur certains gisements, des occupations successives et de courte durée, tout à fait compatibles avec l'utilisation des grottes en bergeries (Léa, à paraître). Ces rythmes sont ainsi tout à fait en accord avec l'hypothèse de la complémentarité entre sites : au Chasséen chaque site est donc bien l'élément d'un réseau.

\section{Silex bédouliens et identité culturelle}

Plusieurs cultures sont contemporaines du Chasséen méridional. Dans les Pyrénéesorientales et la Catalogne, en France comme en Espagne, les cultures des Sepulcres de Fossa et du Montbolo sont définies depuis de nombreuses années à partir des styles céramiques. En revanche, les études lithiques étaient encore inexistantes dans ces contextes chrono-culturels (Léa 1998). J'ai alors décidé d'entreprendre l'analyse technologique des productions lithiques de la grotte de Montou (Corbère-les-Cabanes, Pyrénées-orientales) occupée durant le Montbolo, partiellement contemporain du Chasséen. Mon analyse lithique a ainsi mis en évidence l'importation de préformes et de lames en silex bédouliens vauclusiens (Léa, à paraître). Montou est à l'heure actuelle 
le site le plus occidental sur lequel ces productions ont été identifiées et caractérisées. Les échanges de silex blonds bédouliens, ne s'effectuent donc pas seulement entre les différents groupes du Chasséen méridional, mais sont aussi interculturels. Le franchissement de frontières culturelles, a déjà été remarqué pour différentes productions spécialisées en archéologie, mais c'est ici la première fois que cela est démontré pour les silex bédouliens. Cet échange entre communautés de culture différente, est d'ailleurs pour certains ethnologues-préhistoriens comme Leroi-Gourhan, la condition sine qua non à la survivance d'une innovation sur le long terme, car elle ne se prolongera pas forcément chez les descendants de son inventeur (Leroi-Gourhan 1973, p. 431).

\section{BIBLIOGRAPHIE}

Bazile s.p., BAZILE F., Matières premières minérales et Paléolithique supérieur en Languedoc oriental : une entreprise délicate, in : Actes du colloque « Pierre et archéologie », Tautavel Mai 1998, Perpignan, Presses Universitaires, s.p.

Binder 1984, BINDER D., Systèmes de débitage laminaire par pression : exemples chasséens provençaux, in : Préhistoire de la pierre taillée, 2 : économie du débitage laminaire, Tixier J., Inizan M.L., Roche H. Eds., Paris, Cercle de Recherches et d'Etudes Préhistoriques, 1984, p. 71-84.

Binder 1991, BINDER D., Facteurs de variabilité des outillages lithiques chasséens dans le sud-est de la France, in : Identité du Chasséen : Actes du Colloque international de Nemours, 1989, Beeching A., Binder D., Blanchet J.-C. et al. Eds., Nemours, APRAIF, 1991, p. 261-272 (Mémoire du Musée de Préhistoire d'Ile de France ; 4).

Binder 1996, BINDER D., La villa Giribaldi à Nice : recherches sur un établissement du Néolithique moyen, in : La vie préhistorique, Société préhistorique française Ed., Dijon, Faton, 1996, p. 354-357.

Binder \& Gassin 1988, BINDER D., GASSIN B., Le débitage laminaire chasséen après chauffe : technologie et traces d'utilisation, in : Industries lithiques, tracéologie et technologie, Beyries S. Ed., Oxford, B.A.R., 1988, p. 93-125 (British Archaeological Reports - International Series ; 411).

Binder \& Perlès 1990, BINDER D., PERLÈS C., Stratégie de gestion des outillages lithiques au Néolithique, Paléo, Les Eyzies, 2, 1990, p. 257-283.

Briois 1997, BRIOIS F., Les industries lithiques du Languedoc méditerranéen (6000-2000 av. J.C.). Rythmes et évolution dans la fabrication des outillages de pierre taillée néolithiques entre mer et continent, Ecole des Hautes Etudes en Sciences Sociales, 1997, Thèse de 3ème cycle, 557 p.

Briois \& Léa s.p., BRIOIS F., LÉA V., Productions lithiques autochtones et identité du Chasséen : l'exemple de deux sites de la vallée du Lez (Hérault), in : Actes des 4èmes Rencontres méridionales de Préhistoire récente «Temps et espaces culturels - Actualité de la recherche en Préhistoire récente dans le Midi ", Nîmes, octobre 2000, s.p.

Gassin \& Binder s.p., GASSIN B., BINDER D., avec la collaboration de I. SÉNÉPART, Statut et fonction des productions d'éclats au Néolithique : exemples provençaux, in : Actes du 25ème Congrès préhistorique de France " Approches fonctionnelles en Préhistoire ", Nanterre, novembre 2000, s.p. 
Léa 1997, LÉA V., Contribution à l'étude des sites chasséens de plein air : étude technologique de l'industrie en silex blonds du site de la Cabre (Var), Université de Provence, 1997, Mémoire de Maîtrise, 195 p., 26 fig., 16 pl., 62 tabl.

Léa 1998, LÉA V., Bilan documentaire sur le Chasséen méridional : historiographie et concepts - état de la question - perspectives de recherches lithiques, Université de Provence, 1998, Diplôme d'Etudes approfondies Préhistoire, 193 p.

Léa à paraître, LÉA V., Quelles identités pour l'industrie lithique du Néolithique moyen de Montou ?, in : La grotte de Montou (Pyrénées-Orientales) : étude chronostratigraphique, environnementale et fonctionnelle, Claustre F. Ed., Paris, Maison des Sciences de l'Homme, à paraître (Documents d'Archéologie française).

Leroi-Gourhan 1973, LEROI-GOURHAN A., Milieu et techniques. Réédition, Paris, Albin Michel, 1973, 475 p.

Vaquer 1989, VAQUER J., Innovation et inertie dans le processus de néolithisation en Languedoc occidental, in : Néolithisations, Aurenche O., Cauvin J. Eds., Oxford, B.A.R., 1989, p. 187-198 (British Archaeological Reports - International Series ; 516).

Vaquer 1990, VAQUER J., Le Néolithique en Languedoc occidental, Paris, CNRS, 1990, 397 p.

\section{AUTEUR}

\section{VANESSA LÉA}

